 \\ TATRA \\ MOUNTaiNS \\ Mathematical Publications
}

\section{DYNAMICS OF DARBOUX FUNCTIONS}

\author{
Helena PaWlak — Ryszard J. PaWlak
}

\begin{abstract}
Some results connected with dynamics of quasi-continuous functions or functions possessing a dense orbit and belonging to the class $d$-Conn are presented.
\end{abstract}

\section{Introduction}

A lot of papers which appeared during last few years contain results being a connection with real functions theory and certain elements of dynamical systems, see [5, 10, 11, 12, 13, 15, 16, 17. All aforementioned papers deal with Darboux-like functions. Simultaneously, papers concerning other classes of discontinuous functions have also appeared, e.g., [14, 6]. The last two quoted papers are connected with the theory of chaos understood in different meaning (Devaney, Wiggins, Li and York or Marteli sense). In this paper we hark back to these problems. The second part of this paper (the first part contains preliminaries) is connected with the article [6] in which the authors considered quasicontinuous systems of functions. The natural question connected with this topic is as follows: What kind of assumptions should we additionally impose on quasicontinuous function $f$ in order to obtain that $(f,[0,1])$ be a quasi-continuous system? We will prove that it is sufficient to assume that the considered functions possess the Darboux property. The third part of this paper deals with the entropy and at the same time, in a sense, it harks back to the theory of Li and York chaos [1, p. 232]. We will prove that any function $f \in d$-Conn possessing a dense orbit has a positive entropy.

2000 Mathematics Subject Classification: 26A15, 26A09, 37B40, 26A18, 54H20, $37 \mathrm{E} 15$.

Keyw ords: Darboux function, quasi-continuity, $d$-quasi-continuity, $d$-Conn, entropy, periodic point, turbulent function. 


\section{HELENA PAWLAK - RYSZARD J. PAWLAK}

\section{Preliminaries}

We mostly use standard definitions and notations (see [1, 2, 4, 9]).

We consider only functions mapping unit interval $[0,1]$ into itself. We write $(a, b),[a, b]$ etc. instead of intersections: $(a, b) \cap[0,1],[a, b] \cap[0,1]$ etc. Moreover, for the simplification of the notations, we use symbols $(a, b),[a, b]$ etc. also in the case if $a>b$.

Let $f$ be a function. Then $f^{0}(x)=x$, and $f^{n}(x)=f\left(f^{n-1}(x)\right)$ if $n>0$. A point $x$ such that $f^{M}(x)=x$ but $f^{n}(x) \neq x$ for $n \in\{1,2, \ldots, M-1\}$ is called a periodic point of $f$ of prime period $M$. The set of all periodic points of $f$ of prime period $M$ is denoted by $\operatorname{Per}_{M}(f)$.

The symbol Fix $(f)$ will stand for the set of all fixed points of $f$.

We say [4] that $f:[0,1] \rightarrow[0,1]$ is a Darboux function if for every $x, y \in[0,1]$ and $\alpha$ lying between $f(x)$ and $f(y)$, there is a number $z$ lying between $x$ and $y$ such that $f(z)=\alpha$. By $d$-Conn we will denote the family of all functions $f:[0,1] \longrightarrow[0,1]$ such that $f^{i}$ has a connected graph for $i=1,2, \ldots$ (i.e., any iteration of $f$ has a connected graph). Of course, if $f \in d$-Conn, then $f$ is a Darboux function.

The interior (closure) of a set $A$ (in the space $[0,1]$ with the natural metric) is denoted by $\operatorname{Int}(A)(\bar{A})$.

If $A$ is a subset of the domain of $f$, then $f \uparrow A$ denotes the restriction of $f$ to $A$.

If $f$ is a function, then the set $\gamma_{f}\left(x_{0}\right)=\left\{f^{n}\left(x_{0}\right): n=0,1,2, \ldots\right\}$ is called an orbit of $f$ at $x_{0}$. In this paper we will consider dense orbits of functions. To simplify notations we say "a function $f$ has a dense orbit" in the meaning "there exists a point $x$ such that $\gamma_{f}(x)$ is a dense set" and "a point $x_{0}$ has a dense orbit" instead of " $\gamma_{f}\left(x_{0}\right)$ is a dense set".

A function $f$ is turbulent if there are subintervals $I_{1}, I_{2}$ of $[0,1]$ with at most one point in common such that

$$
I_{1} \cup I_{2} \subset f\left(I_{1}\right) \cap f\left(I_{2}\right) .
$$

Let $f$ be a function, $\varepsilon>0$ and $n$ a positive integer. A set $M \subset[0,1]$ is $(n, \varepsilon)$-separated if for each $x, y \in M, x \neq y$ there is $i(0 \leq i<n)$ such that $\left|f^{i}(x)-f^{i}(y)\right|>\varepsilon$. Let $S(f, n, \varepsilon)$ denote an $(n, \varepsilon)$-separated set with a maximal possible number of points and $s_{n}(\varepsilon)$ its cardinality.

The topological entropy of a function $f$ is a number

$$
h(f)=\lim _{\varepsilon \rightarrow 0} \limsup _{n \rightarrow \infty} \frac{1}{n} \log s_{n}(\varepsilon) .
$$

Our definition coincides with the B owen and Dinaburg version of the topological entropy [3, 8. Note that this is compatible with other definitions of topological entropy [5, 1]. 


\section{DYNAMICS OF DARBOUX FUNCTIONS}

\section{Quasi-continuity and $d$-quasi-continuity}

Let us start with the definition of the quasi-continuity. We say that a function $f$ is quasi-continuous at $x_{0} \in[0,1]$ if for each positive numbers $\varepsilon$ and $\delta$ there exists a nonempty open subset $U$ of $\left(x_{0}-\delta, x_{0}+\delta\right)$ such that

$$
f(U) \subset\left(f\left(x_{0}\right)-\varepsilon, f\left(x_{0}\right)+\varepsilon\right) .
$$

In the paper [6], the quasi-continuous systems of functions have been considered (we say that the system $(f, X)$, where $X$ is some topological space, is quasicontinuous if for every positive integer $n f^{n}: X \longrightarrow X$ is quasi-continuous [as a function]). The authors proved that, for the functions giving quasi-continuous systems, two nonlinear definitions of chaotic dynamical systems are equivalent. Moreover, in the same paper, one can find an example (Example 1.1) of a quasicontinuous function $f$ such that $f^{2}$ is not quasi-continuous. This leads us to the natural question:

What kind of assumptions should be additionally imposed on a quasicontinuous function $f$ in order to obtain that $(f,[0,1])$ is a quasicontinuous system?

Giving an answer to this question, we will obtain stronger results showing that if we additionally assume that a quasi-continuous function is a Darboux function, then a system of functions $\left\{f^{n}: n=1,2, \ldots\right\}$ consists of "uniformly quasi-continuous" functions. So, we will introduce the following definition which presents "uniform quasi-continuity" in the language of dynamical systems.

We say that a function $f$ is d-quasi-continuous at $x_{0} \in[0,1]$ if for every positive numbers $\varepsilon$ and $\delta$ and positive integer $m$ there exists a nonempty open subset $U$ of $\left(x_{0}-\delta, x_{0}+\delta\right)$ such that

$$
f^{i}(U) \subset\left(f^{i}\left(x_{0}\right)-\varepsilon, f^{i}\left(x_{0}\right)+\varepsilon\right) \quad \text { for all } i \in\{1, \ldots, m\} .
$$

If $f$ is a quasi-continuous ( $d$-quasi-continuous) function at every point of the domain, we say that $f$ is a quasi-continuous function (d-quasi-continuous function).

Theorem 2.1. If $f:[0,1] \longrightarrow[0,1]$ is a Darboux and quasi-continuous function, then $f$ is d-quasi-continuous.

P r o of. Let us fix numbers $x_{0} \in[0,1], \varepsilon>0, \delta>0$ and a positive integer $m_{0}$. If there exists $\delta_{1}>0$ such that $f \uparrow\left[x_{0}-\delta_{1}, x_{0}+\delta_{1}\right]$ is a constant function, then it is sufficient to put $U=\left(x_{0}-\min \left\{\delta, \delta_{1}\right\}, x_{0}+\min \left\{\delta, \delta_{1}\right\}\right)$.

So now we will assume that the function $f \uparrow\left[x_{0}-\eta, x_{0}+\eta\right]$ is not constant for any $\eta>0$.

Let $A_{0}=\left[x_{0}-\frac{\delta}{2}, x_{0}+\frac{\delta}{2}\right]$. 


\section{HELENA PAWLAK - RYSZARD J. PAWLAK}

Since $f$ is a Darboux function, then $f\left(A_{0}\right)$ is a nondegenerate interval containing $f\left(x_{0}\right)$. Since $f\left(A_{0}\right) \neq\left\{f\left(x_{0}\right)\right\}$, then there exists $p_{1}$ such that

$$
p_{1} \in\left(f\left(x_{0}\right)-\varepsilon, f\left(x_{0}\right)+\varepsilon\right) \cap\left(f\left(A_{0}\right) \backslash\left\{f\left(x_{0}\right)\right\}\right) .
$$

Let

$$
A_{1}=\left[f\left(x_{0}\right), p_{1}\right]
$$

then

$$
A_{1} \subset f\left(A_{0}\right) \text {. }
$$

Thus, $f\left(A_{1}\right)$ is an interval containing $f^{2}\left(x_{0}\right)$.

If $f\left(A_{1}\right)=\left\{f^{2}\left(x_{0}\right)\right\}$, then we can put $A_{i}=\left\{f^{2}\left(x_{0}\right)\right\}$ for $i=2, \ldots, m_{0}$.

In opposite case (i.e., $f\left(A_{1}\right) \neq\left\{f^{2}\left(x_{0}\right)\right\}$ ), there exists $p_{2}$ such that

$$
p_{2} \in\left(f^{2}\left(x_{0}\right)-\varepsilon, f^{2}\left(x_{0}\right)+\varepsilon\right) \cap\left(f\left(A_{1}\right) \backslash\left\{f^{2}\left(x_{0}\right)\right\}\right) .
$$

In that case let

$$
A_{2}=\left[f^{2}\left(x_{0}\right), p_{2}\right]
$$

then

$$
A_{2}=\left[f^{2}\left(x_{0}\right), p_{2}\right] \subset\left(f^{2}\left(x_{0}\right)-\varepsilon, f^{2}\left(x_{0}\right)+\varepsilon\right) .
$$

In both cases

$$
A_{2} \subset f\left(A_{1}\right) .
$$

Now, considering similarly, if $f\left(A_{2}\right)=\left\{f^{3}\left(x_{0}\right)\right\}$, then we can put

$$
A_{i}=\left\{f^{3}\left(x_{0}\right)\right\} \quad \text { for } \quad i=3, \ldots, m_{0} .
$$

In opposite case there exists

$$
p_{3} \in\left(f^{3}\left(x_{0}\right)-\varepsilon, f^{3}\left(x_{0}\right)+\varepsilon\right) \cap\left(f\left(A_{2}\right) \backslash\left\{f^{3}\left(x_{0}\right)\right\}\right) .
$$

In that case, let

$$
A_{3}=\left[f^{3}\left(x_{0}\right), p_{3}\right],
$$

then

$$
A_{3}=\left[f^{3}\left(x_{0}\right), p_{3}\right] \subset\left(f^{3}\left(x_{0}\right)-\varepsilon, f^{3}\left(x_{0}\right)+\varepsilon\right) .
$$

In both cases,

$$
A_{3} \subset f\left(A_{2}\right) .
$$

Continuing this procedure we can obtain a finite sequence of closed intervals $\left\{A_{i}\right\}_{i=0}^{m_{0}+1}$ such that

$$
A_{0} \subset\left(x_{0}-\delta, x_{0}+\delta\right) \text { and } A_{i} \subset\left(f^{i}\left(x_{0}\right)-\varepsilon, f^{i}\left(x_{0}\right)+\varepsilon\right)
$$

for $i=1,2, \ldots, m_{0}+1$. 


\section{DYNAMICS OF DARBOUX FUNCTIONS}

Let $k_{0}$ be the greatest number belonging to $\left\{0,1, \ldots, m_{0}\right\}$ such that $f\left(A_{k_{0}}\right)$ is a nondegenerate interval. Let us put

$$
M_{0}=\left\{x_{0}, x_{0}-\frac{\delta}{2}, x_{0}+\frac{\delta}{2}\right\} .
$$

If $k_{0}>0$, then let $M_{1}=f\left(M_{0}\right) \cup\left\{p_{1}\right\}$.

If $k_{0}>1$, then let $M_{2}=f\left(M_{1}\right) \cup\left\{p_{2}\right\}$.

Continuing this procedure we obtain a sequence of sets $M_{0}, M_{1}, \ldots, M_{k_{0}}$.

Remark that if $k_{0}<m_{0}$, then $f\left(A_{k_{0}+1}\right)=\left\{f^{k_{0}+2}\left(x_{0}\right)\right\}$.

Since $M_{k_{0}+1}$ is a finite set, then there exists an element $y_{k_{0}+1}$ such that

$$
y_{k_{0}+1} \in A_{k_{0}+1} \backslash M_{k_{0}+1} \subset\left(f^{k_{0}+1}\left(x_{0}\right), p_{k_{0}+1}\right) .
$$

According to the Darboux property of $f$ and the inclusion $M_{k_{0}+1} \supset f\left(M_{k_{0}}\right)$, we can infer that there exists $y_{k_{0}} \in \operatorname{Int}\left(A_{k_{0}}\right) \backslash M_{k_{0}}$ such that $f\left(y_{k_{0}}\right)=y_{k_{0}+1}$.

Since $f$ is quasi-continuous at the point $y_{k_{0}}$, then there exists an open set

$$
W_{k_{0}} \subset\left(f^{k_{0}}\left(x_{0}\right), p_{k_{0}}\right) \backslash M_{k_{0}}
$$

such that

$$
f\left(W_{k_{0}}\right) \subset\left(f^{k_{0}+1}\left(x_{0}\right), p_{k_{0}+1}\right) \subset\left(f^{k_{0}+1}\left(x_{0}\right)-\varepsilon, f^{k_{0}+1}\left(x_{0}\right)+\varepsilon\right) .
$$

Let $z_{k_{0}} \in W_{k_{0}}$. It is easy to see that $f\left(p_{k_{0}-1}\right) \neq z_{k_{0}}\left(z_{k_{0}} \notin f\left(M_{k_{0}-1}\right)\right)$. Then ( $f$ is a Darboux function) there exists an element

$$
y_{k_{0}-1} \in\left(f^{k_{0}-1}\left(x_{0}\right), p_{k_{0}-1}\right) \backslash M_{k_{0}-1}
$$

such that $f\left(y_{k_{0}-1}\right)=z_{k_{0}}$.

Repeating application of the quasi-continuity of $f\left(\right.$ at $\left.y_{k_{0}-1}\right)$, we can deduce that there exists an open set $W_{k_{0}-1} \subset\left(f^{k_{0}-1}\left(x_{0}\right), p_{k_{0}-1}\right) \backslash M_{k_{0}-1}$ such that $f\left(W_{k_{0}-1}\right) \subset W_{k_{0}}$.

Now, if $k_{0}-1 \neq 0$, then let $z_{k_{0}-1} \in W_{k_{0}-1}$. Of course, $f\left(p_{k_{0}-2}\right) \neq z_{k_{0}-1}$. Then there exists an element $y_{k_{0}-2} \in\left(f^{k_{0}-2}\left(x_{0}\right), p_{k_{0}-2}\right) \backslash M_{k_{0}-2}$ such that $f\left(y_{k_{0}-2}\right)=z_{k_{0}-1}$. According to quasi-continuity of $f$ (at $\left.y_{k_{0}-2}\right)$, we can deduce that there exists an open set $W_{k_{0}-2} \subset\left(f^{k_{0}-2}\left(x_{0}\right), p_{k_{0}-2}\right) \backslash M_{k_{0}-2}$ such that $f\left(W_{k_{0}-2}\right) \subset W_{k_{0}-1}$.

Continuing this procedure we obtain a sequence of nonempty open sets $W_{0}$, $W_{1}, \ldots, W_{k_{0}}$ such that

$$
\begin{aligned}
& f\left(W_{0}\right) \subset W_{1} ; \quad f^{2}\left(W_{0}\right) \subset f\left(W_{1}\right) \subset W_{2} ; \ldots ; \\
& f^{k_{0}}\left(W_{0}\right) \subset f\left(f^{k_{0}-1}\left(W_{0}\right)\right) \subset f\left(W_{k_{0}-1}\right) \subset W_{k_{0}}
\end{aligned}
$$

and

$$
f^{i}\left(W_{0}\right) \subset\left(f^{i}\left(x_{0}\right)-\varepsilon, f^{i}\left(x_{0}\right)+\varepsilon\right) \quad \text { for } \quad i=1,2, \ldots, k_{0}
$$


moreover,

$$
W_{0} \subset\left(x_{0}-\delta, x_{0}+\delta\right) .
$$

If $k_{0}=m_{0}$, then the proof is finished.

In case $k_{0}<m_{0}$, we have $f^{k_{0}+1}\left(W_{0}\right)=\left\{f^{k_{0}+1}\left(x_{0}\right)\right\}$ and, consequently,

$$
f^{k_{0}+i}\left(W_{0}\right)=\left\{f^{k_{0}+i}\left(x_{0}\right)\right\} \subset\left(f^{k_{0}+i}\left(x_{0}\right)-\varepsilon, f^{k_{0}+i}\left(x_{0}\right)+\varepsilon\right)
$$

for $i=0,1, \ldots, m_{0}-k_{0}$.

The paper 6] contains a lot of interesting results. Unfortunately, the assumption that $(f, X)$ is quasi-continuous system is rather inconvenient, because there are no tools giving possibility to examine quasi-continuity of compositions of functions. The above theorem gives some solution of this problem. It follows from the above theorem that a lot of interesting results obtained in 6, e.g., Theorem $2.1,2.2,3.1]$ remain true, if we consider Darboux and quasi-continuous functions defined in $[0,1]$.

\section{The entropy}

Theory of transitivity (see [7, 18]) of functions $f:[0,1] \longrightarrow[0,1]$ plays a very important role in the theory of dynamical systems of continuous functions. This is connected with its applications to some considerations in the theory of chaos [7. For example, some kind of chaos is implicated by the transitivity of functions $f:[0,1] \longrightarrow[0,1][18$. On the other hand, it is well-known $[2]$ that for a continuous function $f:[0,1] \longrightarrow[0,1]$ the following equivalence is true: $f$ is a transitive function if and only if it possesses a dense orbit. In case of discontinuous functions there is no such relation (e.g., it has been proved in the paper 14 that a transitive map with two points of discontinuity does not have a dense orbit in general). Consequently, it is interesting to consider the problems connected with properties of discontinuous functions possessing a dense orbit. In this part of the paper we will consider entropy of such kind of functions.

Before proceeding we recall some known statements.

Lemma 3.1 ([13, Proposition 2.4]). If $f$ is turbulent and Darboux function, then $h(f)>0$.

This lemma can also be proved by methods applied in [5, the proof of Proposition 4.2].

Lemma 3.2 ([5, Proposition 3.6]). If $f$ is an arbitrary function, then

$$
h\left(f^{i}\right)=i \cdot h(f) .
$$

We will prove the following theorem: 


\section{DYNAMICS OF DARBOUX FUNCTIONS}

TheOREM 3.3. If a function $f \in d$-Conn possesses a dense orbit, then $h(f)>0$.

P r o of. To the contrary, suppose that $h(f)=0$ and let $x_{0}$ be a point possessing the dense orbit $\gamma_{f}\left(x_{0}\right)=\left\{f^{n}\left(x_{0}\right): n=0,1, \ldots\right\}$.

First, we will show that

$$
\operatorname{Fix}(f) \cap(0,1) \neq \emptyset .
$$

Suppose, to the contrary, that $\operatorname{Fix}(f) \cap(0,1)=\emptyset$. Since $f \in d$-Conn, then there are two possible cases:

- $f(x)<x$ for any $x \in(0,1)$,

- $f(x)>x$ for any $x \in(0,1)$.

First, we will consider the first case: $f(x)<x$ for any $x \in(0,1)$. So, let

$$
n_{0}=\min \left\{n \in\{0,1,2\}: f^{n}\left(x_{0}\right) \in(0,1)\right\}
$$

(let us observe that $x_{0} \in(0,1)$ or $f\left(x_{0}\right) \in(0,1)$ or $\left.f^{2}\left(x_{0}\right) \in(0,1)\right)$.

Let us put $y_{0}=f^{n_{0}}\left(x_{0}\right) \in(0,1)$. According to our supposition, we have $f\left(y_{0}\right)<y_{0}$. Now, we show (by the induction on $n$ ) that our assumptions lead to the inequalities

$$
f^{n}\left(y_{0}\right)<y_{0} \quad \text { for any } n .
$$

Of course, if $n=1$, the inequality (2) is true. Assuming that (2) is true for $n$, we will prove it for $n+1$.

First, one can remark that our assumption $f(x)<x$ for $x \in(0,1)$ and the Darboux property of $f$ imply $f(0)=0$. Consequently, we infer that $f(x) \leq x$ for any $x \in[0,1]$.

Thus

$$
f^{n+1}\left(y_{0}\right)=f\left(f^{n}\left(y_{0}\right)\right) \leq f^{n}\left(y_{0}\right)<y_{0} .
$$

This finishes the proof of (2).

The inequalities (2) contradict to the density of the orbit $\gamma_{f}\left(y_{0}\right)$.

Now, let us consider the second case: $f(x)>x$ for any $x \in(0,1)$. In a similar way, one can obtain a contradiction. Consequently, our supposition that Fix $(f) \cap$ $(0,1)=\emptyset$ leads to the contradiction and, at the same time, (11) is proved.

So, let us fix a point $z_{0} \in(0,1) \cap \operatorname{Fix}(f)$.

Now, we will show that

$$
f(y) \neq z_{0} \quad \text { for any } \quad y \in\left(0, z_{0}\right) \cup\left(z_{0}, 1\right) .
$$

Suppose, to the contrary, that there exists $y_{1} \in\left(0, z_{0}\right) \cup\left(z_{0}, 1\right)$ such that $f\left(y_{1}\right)=z_{0}$. Let, for instance, $y_{1} \in\left(z_{0}, 1\right)$. Let us observe that one can choose a point $t_{0}$ belonging to $\left(z_{0}, y_{1}\right)$ such that the orbit

$$
\gamma_{t_{0}}=\left\{f^{n}\left(t_{0}\right): n=0,1,2, \ldots\right\}
$$


is a dense set. This means that there exists a positive integer $n_{1}$ such that $f^{n_{1}}\left(t_{0}\right)>y_{1}$. According to the Darboux property of $f^{n_{1}}$ we infer that

$$
\left[z_{0}, t_{0}\right] \cup\left[t_{0}, y_{1}\right] \subset f^{n_{1}}\left(\left[z_{0}, t_{0}\right]\right) \cap f^{n_{1}}\left(\left[t_{0}, y_{1}\right]\right) \text {. }
$$

From Lemma 3.1 we may conclude that $h\left(f^{n_{1}}\right)>0$, and by Lemma 3.2 $h(f)>0$, on contrary to our supposition that $h(f)=0$.

This finishes the proof of (3).

If there exists a point $p \in\left[0, z_{0}\right)$ such that $f(p)<z_{0}$ then, according to (3) and due to the connectedness of the graph of $f$, we conclude that $f\left(\left[0, z_{0}\right)\right) \subset\left[0, z_{0}\right]$, and consequently, the interval $\left[0, z_{0}\right]$ does not contain any point possessing dense orbit. Thus, we obtained a contradiction.

The above considerations lead us to the conclusion that

$$
\overline{f\left(\left[0, z_{0}\right]\right)} \subset\left[z_{0}, 1\right] .
$$

In a similar way, one can prove that

$$
\overline{f\left(\left[z_{0}, 1\right]\right)} \subset\left[0, z_{0}\right] .
$$

Now, we consider the function $\xi=f^{2}$. According to our supposition and Lemma 3.2, $h(\xi)=0$.

Combining (44) and (5), we obtain

$$
\xi^{n}\left(\left[0, z_{0}\right]\right) \subset\left[0, z_{0}\right] \quad \text { for } n=1,2, \ldots
$$

Consequently, one can consider the function $\xi:\left[0, z_{0}\right] \longrightarrow\left[0, z_{0}\right]$. It has the following properties

$$
\xi^{n} \in d \text {-Conn } \quad \text { for } \quad n=1,2, \ldots
$$

and

$$
\xi \text { has a dense orbit. }
$$

Indeed, the relation (6) is obvious. To prove (7), let us fix a positive integer $n_{a}$ such that $f^{n_{a}}\left(x_{0}\right) \in\left[0, z_{0}\right]$. According to (44) and (5), we have $\left(f^{k}\left(f^{n_{a}}\left(x_{0}\right)\right) \neq z_{0}\right.$ for $k=0,1,2, \ldots)$

$$
f^{k}\left(f^{n_{a}}\left(x_{0}\right)\right) \in\left[0, z_{0}\right] \quad \text { if and only if } k \text { is an even number. }
$$

Consequently,

$$
\gamma_{f}\left(f^{n_{a}}\left(x_{0}\right)\right)=\left\{f^{2 n}\left(f^{n_{a}}\left(x_{0}\right)\right): n=0,1,2, \ldots\right\} \quad \text { is a dense set in }\left[0, z_{0}\right] .
$$

This finishes the proof of (7).

In view of (6) and (7), the analogous considerations for $f$ (replacing 1 with $z_{0}$ ) imply that there exists $z_{0}^{\prime} \in \operatorname{Fix}(\xi) \cap\left(0, z_{0}\right)$ such that (in view of $h(\xi)=0$ )

$$
\xi(y) \neq z_{0}^{\prime} \quad \text { for any } y \in\left(0, z_{0}^{\prime}\right) \cup\left(z_{0}^{\prime}, z_{0}\right) \text {. }
$$




\section{DYNAMICS OF DARBOUX FUNCTIONS}

Consequently, we have

$$
\overline{\xi\left(\left[0, z_{0}^{\prime}\right]\right)} \subset\left[z_{0}^{\prime}, z_{0}\right] \text { and } \overline{\xi\left(\left[z_{0}^{\prime}, z_{0}\right]\right)} \subset\left[0, z_{0}^{\prime}\right] .
$$

The last inclusion means that $\xi\left(z_{0}\right) \in\left[0, z_{0}^{\prime}\right]$ which is impossible because of $\xi\left(z_{0}\right)=z_{0}>z_{0}^{\prime}$.

Our assumption that $h(f)=0$ brings us to a contradiction.

The above theorem (and a lot of other considerations in various papers) leads us to a very interesting problem:

Problem. What kind of assumptions should we additionally impose on a function $f:[0,1] \longrightarrow[0,1]$ possessing a connected graph in order to infer that $f^{n}$ has connected graph for any $n=1,2, \ldots$ ?

\section{REFERENCES}

[1] ALSEDÀ, L.-LLIBRE, J.-MISIUREWICZ M.: Combinatorial Dynamics and Entropy in Dimension One, in: Adv. Ser. Nonlinear Dynam., Vol. 5, World Scientific, Singapore, 1993.

[2] BLOCK, L. S.-COPPEL, W. A.: Dynamics in One Dimension, in: Lecture Notes in Math., Vol. 1513, Springer-Verlag, Berlin, 1992.

[3] BOWEN, R.: Entropy for group endomorphism and homogeneous spaces, Trans. Amer. Math. Soc. 153 (1971), 401-414. Errata: Trans. Amer. Math. Soc. 181 (1973), 509-510.

[4] BRUCKNER, A. M.: Differentation of Real Functions, in: Lecture Notes in Math. Vol. 659, Springer-Verlag, Berlin, 1978.

[5] ČIKLOVÁ, M.: Dynamical systems generated by functions with connected $G_{\delta}$ graphs, Real Anal. Exchange 30 (2004/2005), 617-638.

[6] CRANNELL, A.-MARTELLI, M.: Dynamics of quasicontinuous systems, J. Difference Equ. Appl. 6 (2000), 351-361.

[7] DEVAnEY, R. L.: Chaotic Dynamical Systems (2nd ed.), Addison-Wesley Publ. Comp., Redwood City, CA, 1989.

[8] DINABURG, E. I.: The relation between topological entropy and metric entropy, Soviet Mat. Dokl. 11 (1970), 13-16.

[9] ENGELKING, R.: General Topology, PWN-Polish Scientific Publishers, Warszawa, 1977.

[10] KELLUM, K. L.: Iterates of almost continuous functions and Sharkovskii's theorem, Real Anal. Exchange 14 (1988-1989), 420-422.

[11] PAWLAK, H.-PAWLAK, R.: Transitivity, dense orbits and some topologies finer than the natural topology of the unit interval, Tatra Mt. Math. Publ. 35 (2007), 1-12.

[12] PAWLAK, R. J.: On Sharkovsky's property of Darboux functions, Tatra Mt. Math. Publ. 42 (2009) 95-105.

[13] PAWLAK, R. J.: On the entropy of Darboux functions, Colloq. Math. (to appear).

[14] PERIS, A.: Transitivity, dense orbit and discontinuous functions, Bull. Belg. Math. Soc. 6 (1999), 391-394.

[15] STALLINGS, J.: Fixed point theorem for a connectivity maps, Fund. Math. 47 (1959), $249-263$.

[16] SZUCA, P.: Punkty Stałe Odwzorowań Typu Darboux. Doctoral Thesis, Gdańsk, 2003. (In Polish) 


\section{HELENA PAWLAK - RYSZARD J. PAWLAK}

[17] SZUCA, P.: Sharkovskiı's theorem holds for some discontinuous functions, Fund. Math. 179 (2003), 27-41.

[18] VAlLEKOOP, M.-BERGLUND, R.: On intervals, transitivity chaos, Amer. Math. Monthly 101 (1994), 353-353.

Received September 24, 2007

Faculty of Mathematics and Computer Science

Łódź University

Banacha 22

PL-90-238 Łódź

POLAND

E-mail: rpawlak@math.uni.lodz.pl 